\title{
START Learning by Sharing: FAQs
}

\author{
Keith A. Rodvold, PharmD, FCCP, FIDSA
}

A n integral and critical component of the START educational program was discussion pertaining to regional and local issues related to community-acquired pneumonia (CAP) and antimicrobial stewardship. Below are some of the frequently asked questions during the START educational program.

\section{Question 1: Sending samples to an outside laboratory for pathogen identification results in a much lower rate of $\boldsymbol{S}$. pneumoniae than what is expected based on published reports. Is this accurate? How useful are sputum samples in determining the causative pathogen in CAP patients?}

Response: S. pneumoniae is the most common causative pathogen associated with mild and severe cases of CAP. ${ }^{1}$ S. pneumoniae is not a robust organism and does not survive for prolonged periods of time under inhospitable conditions. Sending culture samples to an outside microbiology laboratory, which may involve overnight shipment and extreme temperature variations, can result in complete or nearly complete die-off of the S. pneumoniae in that sample. This can lead to inaccurate results. For example, shipping a sample that may contain $10^{6}$ cells of S. pneumoniae along with only a few cells of a more robust organism, such as Staphylococcus aureus or Escherichia coli, may lead to a culture result that only shows the more robust organism though this may be due to contamination of the sample. Therefore, an on-site laboratory should always be preferred in order to limit the delay in culture testing. Rapid methods of detecting organisms in sputum samples, such as polymerase chain reaction (PCR), can be used as a diagnostic tool or to confirm microbiological results.

\section{Question 2: How effective is the pneumococcal vaccine in preventing infection?}

Response: Currently, the 2 commonly used pneumococcal vaccines are the 7-valent vaccine administered to children and the 23 -valent vaccine administered to adults. Both vaccines work well in preventing invasive infections, such as bacteremia and meningitis, caused by strains covered in the vaccine. Recent reports have also shown a benefit of the vaccine in reducing the incidence of noninvasive infections, such as pneumococcal pneumonia, in children and older adults. ${ }^{2,3}$

As a consequence of the use of the pneumococcal vaccine, there has been an apparent shift in the predominant genotypes of S. pneumoniae strains, such as serotype 19A. ${ }^{4}$ The prevalence of strains that are covered in the vaccine and were previously major causes of infection has reportedly decreased, while strains not covered in the vaccine have become more common. As a result, the pneumococcal vaccine will need to be updated periodically to ensure that the predominant strains causing infection are covered.

\section{Question 3: As a result of the passage of the Pharmacist Immunization Bill in the New York State, how many com- munity pharmacists will actually be involved in immunizing patients against influenza?}

Response: With the passage of the Pharmacist Immunization Bill, New York joins 48 other states that allow pharmacists to adminis- ter influenza and pneumococcal vaccines. This can help alleviate the strain on primary care facilities and hospitals particularly at the start of the flu season when the demand for these vaccinations is very high. States that allow pharmacists to administer vaccinations have seen an increase in influenza vaccination rates. One study compared influenza vaccination rates in states that allow pharmacists to administer vaccinations versus states that do not. ${ }^{5}$ Where pharmacists were allowed to administer vaccinations, the vaccination rate increased from $58 \%$ in 1995 to $68 \%$ in 1999 , compared to an increase of $61 \%$ to $65 \%$ in states that do not allow pharmacists to administer vaccines. However, due to the legal implications of pharmacists administering injections, many community pharmacy chains may not allow their pharmacists to actually perform the vaccinations but may hire qualified nurses to perform this duty.

Question 4: There has been a growing concern regarding methicillin-resistant $S$. aureus (MRSA) infections over the past few years. Is MRSA implicated in CAP or is it predominantly associated with skin and soft tissue infections?

Response: Sixty percent of $S$. aureus isolates in the hospitals are methicillin-resistant, but this percentage can vary greatly depending on geographic location or the institution. ${ }^{6}$ Historically, MRSA has been confined to the hospital setting as a major cause of nosocomial infections, particularly skin and soft tissue infections and ventilator-associated pneumonia. However, we are now seeing more patients who acquire a MRSA infection in the community. Earlier, the isolates responsible for hospital-acquired MRSA infections and community-acquired MRSA infections could be differentiated based on their genetic elements, patient characteristics, and antimicrobial resistance profiles. However, this is no longer the case as hospitalized patients are being identified with MRSA strains that are typically associated with community-acquired infections, and vice versa. ${ }^{7}$ This has complicated diagnoses and hence appropriate therapy. Among CAP patients, MRSA is not a predominant cause of infection. However, as the prevalence of MRSA increases in the community, we may see an increase in the incidence of necrotizing pneumonia due to MRSA.

\section{Question 5: What are the risk factors for CAP due to MRSA?}

Response: Providing pathogen-directed therapy upfront is important; therefore, recognizing risk factors for potential pathogens is critical. The 3 major risk factors for CA-MRSA pneumonia are (a) positive Gram stain, (b) influenza-like symptoms, and (c) previous antimicrobial use. ${ }^{8}$ In cases of pneumonia caused by MRSA, vancomycin or linezolid are the currently recommended agents. Linezolid is preferred to vancomycin by some clinicians, particularly in areas where the minimum inhibitory concentration (MIC) of $S$. aureus strains is $>1 \mu \mathrm{g}$ per $\mathrm{mL}$ for vancomycin as infections due to these strains have been associated with a greater risk of failure when treated with vancomycin. ${ }^{9}$

Question 6: What are the contraindications for moxifloxacin and other fluoroquinolones?

Response: The contraindications, listed on the package insert, 
include hypersensitivity to moxifloxacin or any other fluoroquinolone. Also, recently, concern associated with QTc interval prolongation with fluoroquinolone use, particularly IV moxifloxacin, has come to the forefront. Patients with heart disorders, cardiac arrhythmias, or taking other medications that may prolong the QTc interval should be prescribed fluoroquinolones with caution. The package insert of all fluoroquinolones has also recently been changed to reflect the risk of tendinitis and tendon rupture with fluoroquinolone use, and clinicians should be made aware of this.

\section{Question 7: As an antimicrobial stewardship tactic, how is antimicrobial pre-authorization implemented at a hospital?}

Response: There are various approaches to implementing an antimicrobial pre-authorization program at a hospital. One approach involves the antimicrobial stewardship program (ASP) team deciding the agents that should be restricted and the approved uses for those agents. A qualified member of the ASP team must be available to respond when an order for a restricted antimicrobial is submitted to the pharmacy. When an order for a restricted drug is submitted to the pharmacy, the pharmacist on duty would contact the ASP personnel and provide details of the patient. The ASP personnel would then approve or disapprove the order. If the order is not approved, the prescribing physician would be contacted immediately for a discussion regarding the decisionmaking process and the reasons for inappropriateness of the agent requested and the alternative agents. All attempts should be made to come to a consensus regarding the antimicrobial to be used. In cases where an agreement cannot be reached, the infectious disease consult service should be approached and asked to determine the authorization of the requested antimicrobial. In cases where the ASP team is not available 24 hours a day, any drug should be released for the first 24-hour treatment, but the use of a restricted drug should be reviewed at the earliest opportunity and must be approved by the ASP team for continuation.

\section{Question 8: In the current ATS/IDSA CAP guidelines, for patients in the ICU not at risk of infection due to Pseudomonas, it seems that double gram-negative coverage is recommended with a $\beta$-lactam plus a fluoroquinolone. Is this necessary?}

Response: The ATS/IDSA guidelines do not promote double gram-negative coverage as a means to provide synergy or attack the pathogen via multiple routes. ${ }^{8}$ ICU patients are extremely ill and may not be able to survive if given antimicrobials with initial inadequate coverage within the first 24 hours. The combination therapy with double gram-negative coverage is to ensure adequate coverage early during the infection and before culture results are available. Once the pathogen has been identified and susceptibility results obtained, it may be possible to de-escalate therapy in order to limit overuse of antimicrobials and to reduce the risk of the emergence of resistance. However, it can be difficult to convince clinicians to change the course of therapy while a patient is doing well, though evidence shows that de-escalation does not impact clinical outcomes and decreases the risk of resistance development. ${ }^{10}$
Question 9: What is the role of infection control in antimicrobial stewardship programs?

Response: The focus on responsible use of antimicrobials has traditionally been in order to preserve their utility and reduce the emergence of resistance. Today, the appropriate use of antimicrobials has also become necessary, since antimicrobial use is a major risk factor for Clostridium difficile infection (CDI) — a major problem in many hospitals and associated with severe morbidity and high mortality. ${ }^{11}$ In the province of Ontario, Canada, where C. difficile has become a serious threat, a new policy requires mandatory reporting of all CDI cases. As a result, hospitals will need to ensure that not only are antimicrobials used responsibly but also infection control, including environmental cleaning, is effective in reducing the spread of this pathogen.

An antimicrobial stewardship program that promotes the appropriate use of antimicrobials along with effective infection control can be critical in protecting patients from CDI and other nosocomial infections. When implementing an antimicrobial stewardship program at an institution, it is therefore helpful to include an infection control officer for important insight and support—a recommendation of the IDSA/SHEA antimicrobial stewardship guidelines.

\section{Question 10: Is routine surveillance of hospital personnel recommended as a tactic to reduce the risk of spreading nosocomial pathogens?}

Response: Surveillance of personnel may be useful only in isolated instances where there is a cluster of infections at a particular institution or medical ward caused by the same strain, such as MRSA. The medical literature has reports of serious infections inadvertently caused by health care personnel. One investigation of a MRSA outbreak in pediatric and neonatal intensive care units found a high carriage rate of MRSA among health care personnel. A policy of strict handwashing and monitoring, as well as periodic and routine active surveillance of cultures as part of infection control measures was suggested in this case. ${ }^{12}$ In the absence of sporadic clusters of infection in hospital wards, it may not be useful to perform routine testing of personnel.

\section{Question 11: Should reducing adverse events be a more prominent goal of antimicrobial stewardship and what meth- ods should be used for detecting or accounting for these events?}

Response: Adverse events, beyond antimicrobial resistance, are an important aspect of therapy that should be better quantified. There is a lack of a good source that illustrates the prevalence of adverse events in addition to what is reported on product information sheets. Many of the adverse events associated with antimicrobials are due to dosing and allergic reactions-majority of these are preventable, and clinicians should work to minimize such risks. Computer-assisted programs instituted at hospitals may help reduce the incidence of adverse events related to antimicrobial use. 


\section{Author}

KEITH A. RODVOLD, PharmD, FCCP, FIDSA, is Professor of Pharmacy Practice and Medicine, Colleges of Pharmacy and Medicine, University of Illinois at Chicago, Chicago, Illinois.

CORRESPONDENCE: Keith A. Rodvold, PharmD, FCCP, FIDSA, $\mathrm{m} / \mathrm{c}$ 886, University of Illinois at Chicago, College of Pharmacy, Room \#164, 833 South Wood Street, Chicago, IL 60612. Tel.: 312.996.3341; Fax: 312.413.1797; E-mail: kar@uic.edu

\section{DISCLOSURES}

Keith A. Rodvold serves as a consultant to Johnson \& Johnson, Astellas, GlaxoSmithKline, Theravance, Targanta, and Intranasal Therapeutics. He is on the advisory committees of Johnson \& Johnson, Targanta, Baxter, and Pfizer and is a member of the speakers' bureaus for Johnson \& Johnson, Wyeth, Pfizer, and Schering-Plough.

Marco P. Cicero, PhD, of Vemco MedEd, LLC, contributed medical writing and editorial assistance. This article is being published as part of a supplement to the START continuing education program for pharmacists and physicians. It is supported by an educational grant from Schering-Plough Corporation.

\section{REFERENCES}

1. File TM. Community-acquired pneumonia. Lancet. 2003;362(9400):19912001.

2. Vila-Corcoles A, Salsench E, Rodriguez-Blanco T, et al. Clinical effectiveness of 23-valent pneumococcal polysaccharide vaccine against pneumonia in middle-aged and older adults: a matched case-control study. Vaccine. 2009 [Epub ahead of print. PMID: 19171174].
3. Centers for Disease Control and Prevention (CDC). Pneumonia hospitalizations among young children before and after introduction of pneumococcal conjugate vaccine - United States, 1997-2006. MMWR Morb Mortal Wkly Rep. 2009;58(1):1-4.

4. Moore MR, Gertz RE, Jr., Woodbury RL, et al. Population snapshot of emergent Streptococcus pneumoniae serotype 19A in the United States, 2005. J Infect Dis. 2008;197(7):1016-27.

5. Steyer TE, Ragucci KR, Pearson WS, Mainous AG. The role of pharmacists in the delivery of influenza vaccinations. Vaccine. 2004;23(3):279.

6. Styers D, Sheehan DJ, Hogan P, Sahm DF. Laboratory-based surveillance of current antimicrobial resistance patterns and trends among Staphylococcus aureus: 2005 status in the United States. Ann Clin Microbiol Antimicrob. 2006;5:2.

7. Klevens RM, Morrison MA, Fridkin SK, et al. Community-associated methicillin-resistant Staphylococcus aureus and healthcare risk factors. Emerg Infect Dis. 2006;12(12):1991-93.

8. Mandell LA, Wunderink RG, Anzueto A, et al.; Infectious Diseases Society of America and American Thoracic Society. Infectious Diseases Society of America/American Thoracic Society consensus guidelines on the management of community-acquired pneumonia in adults. Clin Infect Dis. 2007:44(Suppl 2):S27-S72.

9. Sakoulas G, Moise-Broder PA, Schentag J, Forrest A, Moellering RC, Jr., Eliopoulos GM. Relationship of MIC and bactericidal activity to efficacy of vancomycin for treatment of methicillin-resistant Staphylococcus aureus bacteremia. J Clin Microbiol. 2004:42(6):2398-402.

10. Soo Hoo GW, Wen YE, Nguyen TV, Goetz MB. Impact of clinical guidelines in the management of severe hospital-acquired pneumonia. Chest. 2005;128(4):2778-87.

11. Owens RC, Jr., Donskey CJ, Gaynes RP, Loo VG, Muto CA. Antimicrobial-associated risk factors for Clostridium difficile infection. Clin Infect Dis. 2008;46(Suppl 1):S19-S31.

12. Lin YC, Lauderdale TL, Lin HM, et al. An outbreak of methicillin-resistant Staphylococcus aureus infection in patients of a pediatric intensive care unit and high carriage rate among health care workers. J Microbiol Immunol Infect. 2007;40(4):325-34. 